\title{
DUST AROUND YOUNG STARS: \\ HOW RELATED TO SOLAR SYSTEM DUST?
}

Summary by: M.S. HANNER 


\title{
DUST AROUND YOUNG STARS: HOW RELATED TO SOLAR SYSTEM DUST?
}

\author{
Summary of Joint Discussion \#17 \\ IAU General Assembly, The Hague \\ Martha S. Hanner \\ Jet Propulsion Laboratory \\ California Institute of Technology \\ Pasadena CA 91109
}

\section{INTRODUCTION}

Study of the dust in circumstellar disks around young stars is currently an extremely active area in astronomy. There is little doubt that accretion disks are a natural part of protostellar evolution. Much recent observational and theoretical work is giving us a clearer picture of the physical conditions in dust disks and their evolutionary progression. IRAS observations revealed that many main-sequence stars, such as $\beta$ Pictoris, have circumstellar disks. But whether these disks are related to planetary formation is not yet understood.

A portion of the dust in disks around young stars ultimately may be incorporated into planetary systems. Thus, study of the dust in our own solar system complements the remote sensing of protostellar regions and aids in reconstructing the evolutionary history of the dust. Since comets formed in the cold outer regions of the solar nebula, they may contain intact interstellar grains. As the comets lose material during passage through the warm inner solar system, some of these grains will be released into interplanetary space. Technical advances now allow analysis of individual micrometer or smaller grains in interplanetary dust particles and primitive meteorite samples. Isotopic anomalies and patterns of crystal growth in these particles are yielding tantalizing clues about the interstellar material incorporated into these solar system samples.

Recent studies of interstellar dust and gas phase depletions provide 
information about the characteristics of carbon and silicate grains and other grain components in various environments. The short time scale for grain destruction in the diffuse interstellar medium means that substantial grain growth occurs in dense clouds. Laboratory experiments on lowtemperature ices are improving our understanding of the chemical processes in grain mantles and the formation of organic material.

This Joint Discussion was initiated by Commission 21 Light of the Night Sky and Commission 34 Interstellar Matter in order to tie together these new discoveries and explore the evolution of dust from the interstellar medium to the solar system. The Joint Discussion consisted of invited reviews, listed in the Acknowledgements, and contributed poster papers. Rather than publish the separate papers, it was decided to prepare a summary paper that would tie together the various topics presented, with emphasis on recent progress in each field.

\section{DUST DISKS AROUND YOUNG STARS}

\subsection{Protoplanetary Disks Around Pre-Main Sequence Objects}

Our understanding of the way the solar nebula evolved and the environment experienced by dust grains during the sun's formation rests, in part, on the study of the nature and evolution of young stellar objects (YSOs). The pre-main sequence $\mathrm{T}$ Tauri stars are thought to be the progenitors of solar mass stars. The Herbig Ae/Be stars are more massive and are the likely progenitors of A-type stars, including those with circumstellar disks such as $\alpha$ Lyr and $\beta$ Pic.

Since YSOs form out of the gas and dust in dense molecular clouds, it is not surprising that many exhibit an infrared excess, indicating the presence of warm dust. Many low mass YSOs have been observed in the $\rho$ Ophiuchi cloud core and in the Taurus-Auriga cloud complex. These infrared sources can be classified according to the spectral energy distribution of the infrared excess. Lada (1987) defined the spectral index $\alpha=\operatorname{d} \log \lambda \mathrm{F}_{\lambda} / \mathrm{d} \log \lambda$ between 2.2 and 10 or $25 \mu \mathrm{m}$. Larger $\alpha$ corresponds to cooler dust. The YSOs can be divided into three classes based on $\alpha$ : Class I: $\alpha>0$; Class II: $-1.5<\alpha<0$; Class III: $\alpha<-1.5$ (Lada 1987; Wilking, Lada \& Young 1989; André \& Montmerle 1994). An additional 
Class 0 has been introduced by André et al. (1993) for those objects detected solely at millimeter wavelengths.

Adams, Lada \& Shu (1987) have interpreted the infrared spectral classes as an evolutionary sequence and have modeled the stages for a low-mass protostar. In their models, Class I objects, with spectral energy distribution rising toward longer wavelength, correspond to the infall phase. The protostar and an accretion disk are embedded in an optically thick infalling dust envelope. The infrared flux arises from the spherical dust envelope. Class II objects are visible protostars with nebular disks. These are classical $T$ Tauri stars. The near-infrared maximum is the reddened stellar photosphere, while the infrared excess above the reddened blackbody is thermal emission from dust in the optically thick disk, heated by the central star. Objects with flat spectra $(\alpha=0)$ and objects exhibiting double peaks, one at 1-2.5 $\mu \mathrm{m}$ and one at $\lambda>10 \mu \mathrm{m}$, represent T Tauri stars with disks and remnant infalling envelopes that contribute to the long-wavelength flux. The disk is passive (lack of hot dust) in the double-peaked sources, whereas sources with flat spectra require hotter, more massive accreting disks to generate the $3-5 \mu \mathrm{m}$ flux. Class III objects, with small, if any, infrared excess, are the weak-lined or "naked" $\mathrm{T}$ Tauri stars with a remnant optically thin disk.

If we are seeking precursors of solar system formation, i.e. protoplanetary disks, then we need to ask:

1. What is the evidence for disks around YSOs?

2. How common are disks?

3. Are the disk masses sufficient for the formation of a planetary system?

There are several indirect arguments for the existence of disks around $\mathrm{T}$ Tauri stars:

1. Theoretical: Disks are the natural outcome of the collapse of a rotating cloud core (Tereby, Shu \& Cassen 1984).

2. Blue-shifted emission lines: The [O I] and [S II] forbidden emission lines from the mass-outflow regions in a number of $\mathrm{T}$ Tauri stars are blue-shifted only. This is consistent with the occultation of the receding, red-shifted wind by a disk $\sim 100 \mathrm{AU}$ in size (Appenzeller et al. 1984; Edwards et al. 1987).

3. Polarization: Maps of the polarization around YSOs can be 
explained by multiple scattering with an optically thick disk (Bastien \& Menard 1988, 1990).

4. Extinction: A large amount of circumstellar material around some optically visible stars is inconsistent with spherical geometry (Beckwith et al. 1990).

Direct evidence for disks has been difficult to acquire, because the size of the disks is too small to resolve optically from ground-based telescopes. One candidate object is HL Tau. Although the $2.7 \mathrm{~mm}{ }^{13} \mathrm{CO}$ maps of HL Tau show a disk-like structure (Sargent \& Beckwith 1987, 1991; Hayashi et al. 1993) the emitting region is complex. The gas motions are apparently more complex than the pure Keplerian rotation originally proposed by Sargent \& Beckwith, and probably include infalling and/or outflowing material from a disk-shaped remnant envelope (Hayashi et al.). The flat infrared spectral energy distribution is consistent with the presence of a remnant envelope around this source.

Images from the refurbished Hubble Space Telescope have resolved circumstellar material surrounding 56 out of 110 young stars visible in images of the Orion Nebula (O'Dell \& Wen 1994). Most of the objects are seen in line emission, excited by ultraviolet radiation from the hot young star $\theta^{1} \mathrm{C}$ Ori. The circumstellar material is optically thick enough to obscure background emission from the Orion nebula, yet the central star is not appreciably obscured. Hence, we are most likely seeing disks of material, not spherical envelopes. One source is seen as a dark elliptical disk silhouetted against the bright nebula. O'Dell \& Wen estimate lower limits to the disk masses of $\sim 2 \times 10^{28} \mathrm{~g}$, or $\sim 10^{-5}$ solar masses. The minimum mass of the solar nebula necessary to form the solar system is on the order of $0.01 \mathrm{M}_{0}$. Thus, a better mass estimate of the Orion sources is needed before one can say that the disks are protoplanetary.

Estimating the total mass of dust in the disks is not possible from the infrared fluxes, because the sources are optically thick in the infrared. Moreover, only warm dust will be probed in the mid-infrared. At millimeter wavelengths, however, the disks are optically thin and cold dust can be detected, allowing the dust mass to be estimated from the observed fluxes. Two recent $1.3 \mathrm{~mm}$ surveys have provided statistics on the mass of circumstellar material. Beckwith et al. (1990) surveyed 86 T Tauri stars in the Taurus-Auriga region, including 20 weak-lined or "naked" $T$ Tauri stars. Their survey was sensitive enough to detect $\sim 10^{-4} \mathrm{M}_{\mathrm{o}}$ of dust, 
corresponding to a total mass of $\sim 0.01 \mathrm{M}_{0}$, assuming gas/dust mass ratio of 100 .

André \& Montmerle (1994) observed over 100 YSOs in the $\rho$ Ophiuchi cloud. Among the 46 positive detections, $70-90 \%$ of Class I, $60 \%$ of Class II, and $<15 \%$ of Class III sources were detected. The detection statistics are similar to those of Beckwith et al. when similar definitions of Class II and III are applied. Among sources detected, there is no correlation between peak $1.3 \mathrm{~mm}$ flux and spectral index $\alpha$. Maps of the sources are consistent with the presence of unresolved disks in Class II sources and more extended envelopes in Class I protostars. The average integrated fluxes are a factor of 2 - 3 higher for Class I than for Class II sources. Thus, the amount of dust is not drastically different between Class I and Class II; it is the spatial distribution that differs.

Similar detection statistics were obtained by Henning et al. (1993) in the Chamaelon dark clouds, where $44 \%$ of the 36 objects were detected.

If we assume that the circumstellar dust is optically thin at $1.3 \mathrm{~mm}$, the mass is given by $M=S_{v} d^{2} /\left(\mathrm{kB}_{v}(\mathrm{~T})\right)$, where $S_{v}$ is the $1.3 \mathrm{~mm}$ integrated flux, $d$ is the distance to the source, $B_{v}(T)$ is the Planck function for dust temperature $T$ and $k$ is the mass absorption coefficient. The largest uncertainty in the mass estimate is the value of the dust mass absorption coefficient. Beckwith et al. adopted $\mathrm{k}=2 \mathrm{~cm}^{2} / \mathrm{g}$ of dust, based on $\mathrm{\kappa} \propto \lambda^{-1}$ (Beckwith \& Sargent 1991), instead of the lower values often assumed based on $\kappa \propto \lambda^{-2}$ (e.g. Draine \& Lee 1984; Hildebrand 1983). A value of $\sim 1.8 \mathrm{~cm}^{2} / \mathrm{g}$ has subsequently been measured in the laboratory for amorphous silicate (forsterite) at $1 \mathrm{~mm}$ (Agladze et al. 1994). A gas/dust mass ratio of 100 is assumed to compute the total mass of material. The average disk mass in the 36 sources detected by Beckwith et al. was $\sim 0.02$ $\mathrm{M}_{\mathrm{o}}$, comparable to the minimum mass required for the solar nebula. Beckwith et al. find no correlation between disk mass and age from $10^{5}$ $10^{7}$ years. The most massive disks are not associated with the youngest stars. Stars in their sample with close companions have little dust mass; more distant companions apparently have little influence on the disk mass.

Using $\mathrm{\kappa}=2 \mathrm{~cm}^{2} / \mathrm{g}$ of dust, André \& Montmerle derive total masses ranging from $0.002-0.1 \mathrm{M}_{\mathrm{o}}$ for Class II sources, with median $\sim 0.01 \mathrm{M}_{\mathrm{o}}$ for detected sources. In other words, about half of the $60 \%$ detected, or $\sim 30 \%$ of classical T Tauri stars, have disk masses above the minimum solar nebula mass of $0.01 \mathrm{M}_{0}$; only these can truly be classified as protoplanetary 
disks. For Class I sources, André \& Montmerle find masses from 0.015 $0.15 \mathrm{M}_{0}$, based on $\mathrm{\kappa}=1 \mathrm{~cm}^{2} / \mathrm{g}$ of dust, with median mass $0.06 \mathrm{M}_{\mathrm{o}}$. The significance of this result is that the masses of Class I envelopes are low, much less than the presumed mass of the central protostar. Thus, these Class I objects cannot represent the main infall stage as modeled by Adams et al. (1987). Only the Class 0 objects, which have strong millimeter fluxes but are invisible in the mid-infrared, have masses $\sim 0.5 \mathrm{M}_{o}$, implying that these objects are in the main accretionary phase. From Class 0 to Class III, then, there is a systematic decrease in circumstellar (disk + envelope) mass.

The best candidate for a more massive disk is the object IRS5, a deeply embedded YSO with a strong bipolar molecular outflow in the dark cloud L1551. Keene \& Mason (1990) observed the thermal continuum radiation at 1.25 and $2.7 \mathrm{~mm}$. Their observations are consistent with a two-component model of a power-law envelope and a compact disk having a radius of $45 \pm 20$ AU. Keene \& Mason derive a disk mass of $\sim 0.6 \mathrm{M}_{0}$. However, their mass estimate is based on the opacity law of Hildebrand (1983), with $\kappa \propto \lambda^{-2}$. The Beckwith, et al. opacity is a factor of 10 higher at $2.7 \mathrm{~mm}$. One cannot exclude that a substantial fraction of the inner disk is optically thick even at $2.7 \mathrm{~mm}$. Thus, a disk mass of $\sim 0.1 \mathrm{M}_{\mathrm{o}}$ seems a reasonable estimate.

Sources with circumstellar mass $>0.01 \mathrm{M}_{o}$ will be optically thick at $\lambda<100 \mu \mathrm{m}$. The minimum infrared excess for an optically thick disk can be calculated as a function of $L_{*}$. Thus, the observed infrared excess above the reddened photosphere of $\mathrm{T}$ Tauri stars can be used to determine the frequency of optically thick disks with $\mathrm{M}>0.01 \mathrm{M}_{\mathrm{o}}$ (Skrutskie et al. 1990; Strom et al. 1993). These authors find that 30\%-50\% of young $\left(<3 \times 10^{6} \mathrm{yr}\right)$ stars with $\mathrm{M}_{*}<3 \mathrm{M}_{\mathrm{o}}$ have infrared excesses indicative of optically thick disks. Almost no optically thick disks are found for stellar age $\geq 10^{7} \mathrm{yr}$. Sensitive near-infrared photometry has revealed small infrared excesses from optically thin disks around some Class III T Tauri stars (Strom et al. 1993). The lack of energetic winds, blue veiling, and UV excesses indicate that accretion has ceased in these objects.

How rapidly do disks clear? Very few sources have been found with infrared excess intermediate between optically thick and optically thin excesses. Thus, disk clearing must proceed rapidly. Skrutskie et al. give a time scale of $\sim 3 \times 10^{5} \mathrm{yr}$. The three transition objects identified by 
Skrutskie et al. (V819 Tau, DI Tau, and SA076411A) have negligible excess at $\lambda \leq 10 \mu \mathrm{m}$, but do show a far-infrared excess. Apparently, the warm dust at $r<0.2 \mathrm{AU}$ from the central star has been removed, leaving an "inner hole", while cold dust remains at larger distances. What causes the "inner hole"? It is tempting to speculate that planetary formation plays a role in sweeping up material and preventing inward diffusion of dust from larger distance.

In summary, there is substantial evidence that disks surround a sizeable fraction of low-mass YSOs during at least part of their evolution. The infrared spectral energy distribution can distinguish between optically thick disks (Class II, classical T Tauri stars) and optically thin disks (Class III, weak T Tauri stars). However, millimeter surveys suggest that only about $30 \%$ of classical $T$ Tauri stars have protoplanetary disks; that is, disks more massive than the minimum solar nebula mass of $\sim 0.01 \mathrm{M}_{\mathrm{o}}$. The time scale for disk dissipation is short, on the order of $10^{5}$ years. Massive disks are apparently rare (Tereby et al. 1993).

Some of the open problems regarding evolution of circumstellar disks are:

1. Does the circumstellar evolution of YSOs depend on initial conditions?

2. What is the influence of companion stars?

3. What is the influence of magnetic fields?

4. Do Herbig Ae/Be stars have disks?

5. How is disk clearing related to planetary formation?

\subsection{The Dust in $\beta$ Pictoris Systems}

The IRAS satellite detected far-infrared emission surrounding three nearby main sequence A stars, $\alpha$ Lyr, $\alpha$ PsA, and $\beta$ Pic, indicating that these stars are surrounded by solid grains at temperature $\mathrm{T} \sim 100 \mathrm{~K}$. (Backman \& Paresce 1992 and refs. therein). The bolometric luminosity of the excess relative to the stellar luminosity is $2 \times 10^{-5}$ ( $\alpha$ Lyr), $8 \times 10^{-5}$ $\left(\alpha\right.$ PsA) and $3 \times 10^{-3}(\beta$ Pic). Subsequently, about 100 main sequence stars of all spectral types with similar infrared excesses have been found in the IRAS database. A coronographic image of $\beta$ Pic at $\lambda 0.89 \mu \mathrm{m}$ clearly showed an edge-on disk of material extending to $1000 \mathrm{AU}$ from the central 
star (Smith \& Terrile 1984,1987). The infrared brightness contours, thermal models, and stellar rotational velocity are consistent with an equatorial disk seen pole-on for $\alpha \mathrm{Lyr}$ and an intermediate inclination for $\alpha$ PsA.

The spectral energy distribution of the infrared excess depends on the temperature distribution and emissivity $\epsilon(\lambda)$ of the grains in the disk. Since the grains cannot radiate efficiently at wavelengths much greater than their size, grains whose size is much smaller than the wavelength of peak emission for a blackbody in thermal equilibrium will be hotter than larger grains. Computed thermal models for assumed size distributions and spatial distributions compared with the IRAS fluxes lead to characteristic grain sizes of $\sim 100 \mu \mathrm{m}$ for $\alpha \mathrm{Lyr}$, a few tens of micrometers for $\alpha$ PsA, and $\sim 1 \mu \mathrm{m}$ for $\beta$ Pic. The discovery of a silicate emission feature in $\beta$ Pic is further evidence for micrometer grains in the disk (Knacke et al. 1993).

Is there a large amount of cold dust in an outer disk, too cold to have been detected by IRAS? The flux at submillimeter wavelengths can answer this question. Maps of the 3 stars at $800 \mu \mathrm{m}$ show that the disk sizes are comparable at $100 \mu \mathrm{m}$ and $800 \mu \mathrm{m}$ (Zuckerman \& Becklin 1993). The $800 \mu \mathrm{m}$ fluxes fall a factor of 4-10 below a blackbody fit to the 25,60 , and $100 \mu \mathrm{m}$ fluxes. Thus, massive outer disks are not present in these objects. Zuckerman \& Becklin derive minimum disk masses on the order of 0.01 Earth masses for $\alpha$ Lyr and $\alpha$ PsA and $\sim 0.1$ Earth mass for $\beta$ Pic.

The minimum wavelength of excess flux implies a lack of hot grains in these systems. Therefore, the inner region around the central star must have a much lower density of material. The extent of this region in $\alpha$ Lyr, $\alpha$ PsA, and $\beta$ Pic is comparable to the planetary region of the solar system. Since Poynting-Robertson drag would tend to fill in this region over time, an efficient removal mechanism is required. Sweep-up by planets is one possible explanation.

The lifetimes of orbiting grains in the disks due to PoyntingRobertson drag and collisions are short compared to the estimated ages of the stars $\left(10^{8}\right.$ years for $\beta$ Pic to $4 \times 10^{8}$ years for $\alpha$ Lyr). Thus, the dust disks in these three systems are not primordial, but must be replenished. Dust release from comets is one plausible source. Transient, highly red-shifted absorption lines seen in spectra of $\beta$ Pic have been interpreted as the breakup of comets falling into the central star (Beust et al. 1990).

How do the dust disks compare with the solar system? The 
bolometric luminosity of the zodiacal dust cloud is $\sim 8 \times 10^{-8} \mathrm{~L}_{0}$, far below the limit of detectability of IRAS. The Kuiper belt is at the heliocentric distance range comparable to the dust disks in $\beta$ Pic systems. Thermal emission from the population of $\sim 10 \mathrm{~km}$ objects is orders of magnitude too low for detection. Whether there is a significant cross section of dust in the Kuiper belt is not known. From Earth, we have not yet been able to detect dust emission from the Kuiper belt because of the strong emission from nearby warm interplanetary dust. But there is sufficient uncertainty in models for the emission of the interplanetary dust cloud at $60 \mu \mathrm{m}$ and 100 $\mu \mathrm{m}$ that emission from a dust disk at $\mathrm{T}<40 \mathrm{~K}$ and $\mathrm{r}>50 \mathrm{AU}$ could be present within the limits of the IRAS fluxes.

In summary, fundamental questions about the disks in $\beta$ Pic systems remain to be answered.

1. Is there an evolutionary connection between circumstellar disks around young stellar objects and the disks in $\beta$ Pic systems?

2. What is the relationship between dust disks and planetary systems? Do the dust disks represent successful planetary formation or failed planetary formation?

3. What is the reservoir for replenishing the dust disks? Are these systems surrounded by a cloud of comets?

4. Are the dust disks analogous to the solar system Kuiper belt? Is there enough dust in the Kuiper belt to be detectable by IRAS?

5. What fraction of main sequence stars are surrounded by dust? Will a more sensitive infrared search yield many more faint sources?

\section{INTERSTELLAR DUST}

\subsection{Properties of Dust in the Diffuse Interstellar Medium}

The physical properties of interstellar (IS) grains are inferred from the IS extinction curve, polarization, scattering of xrays, thermal emission, spectral features, and IS gas depletions compared with cosmic abundances. Laboratory experiments on IS dust analogs can illuminate the physical and chemical processes affecting IS grains and can relate grain optical properties to physical properties. Finally, identification of IS grain 
components in interplanetary dust and primitive meteorites gives a new approach to learning about IS grain properties.

The ultraviolet, optical, and infrared observations lead to a picture of several grain populations. This set should be viewed as a "working hypothesis", not a final answer, for there are still problems and inconsistencies and not all researchers agree with the following list (Greenberg 1989; Mathis \& Whiffen 1989; Rowan-Robinson 1986, 1992; Duley 1987).

1. $a \sim 0.01 \mu \mathrm{m}$ silicate and carbon grains responsible for the farultraviolet extinction rise

2. $a \sim 0.01 \mu \mathrm{m}$ graphitic carbon to produce the $0.2175 \mu \mathrm{m}$ bump

3 . very small grains $(0.0005-0.002 \mu \mathrm{m})$ producing the infrared emission bands and non-equilibrium thermal emission

4. $a \sim 0.12 \mu \mathrm{m}$ grains composed of silicate and carbon giving rise to the visible and near-infrared extinction. The wavelength of maximum polarization, $\lambda \sim 0.55 \mu \mathrm{m}$ in the diffuse IS medium, implies grain radii $a \sim 0.1 \mu \mathrm{m}$.

The strength and ubiquitous presence of the $0.2175 \mu \mathrm{m}$ extinction bump imply that the carrier must be a cosmically abundant element $\{\mathrm{C}$, $\mathrm{Mg}, \mathrm{Si}, \mathrm{Fe}\}$ while the stability of the central wavelength sets severe limits to the particle size. Graphite remains the strongest candidate (e.g. Draine 1989), although other possibilities have been proposed, such as non-graphitic carbon and $\mathrm{OH}^{-}$on small silicate grains (Duley 1987). While carbon grains are expelled from AGB stars as amorphous carbon, small amorphous carbon grains can be annealed to graphite by the temperature excursion following the absorption of a single UV photon. Uncertainty in the appropriate optical constants for graphite near $0.22 \mu \mathrm{m}$ makes it difficult to specify a unique model. For a given set of optical constants, the peak wavelength of the bump shifts with the shape of the grains. Draine (1989) finds that the peak wavelength can be matched by oblate graphite spheroids in the Rayleigh limit, while variations in the width of the feature may be explained by differences in the oblateness distribution of the grains. The average oblateness, however, has to remain constant, making this explanation somewhat contrived. Further calculations of size and shape effects led Draine \& Malhotra (1993) to conclude that observed variations in the bump profile are most likely due to changes in the dielectric properties of the grain material. Mathis (1994) 
proposes that the variable width is due to coatings on graphite grains, with the narrowest features arising from bare graphite grains.

Excess infrared continuum emission and the series of infrared emission bands indicate that there is a population of very small grains, $a \sim 0.001 \mu \mathrm{m}$. Emission bands at $\lambda 3.3,6.2,7.7,8.6$, and $11.25 \mu \mathrm{m}$ are seen in a variety of sources including reflection nebulae, planetary nebulae, and H II regions, all having in common proximity to a strong ultraviolet radiation field. Weaker features and broad emission plateaus are also present in some sources. These bands are attributed to UV excited fluorescence in polycyclic aromatic hydrocarbons (PAHs) existing either as free molecules, PAH clusters, or within larger hydrogenated amorphous carbon particles (Duley \& Williams 1981; Leger \& Puget 1984; Allamandola, Tielens \& Barker 1985) or to aromatic hydrocarbon components in an amorphous material (Sakata et al. 1987). Yet, despite intensive study over the past decade, no specific material has been identified that provides exact spectral matches. It is not even certain whether the carriers are large planar molecules or small three-dimensional grains.

Reflection nebulae exhibit strong infrared continuum emission, far higher than can be explained by emission from grains in thermal equilibrium. The $1-5 \mu \mathrm{m}$ color temperature of $\sim 1000 \mathrm{~K}$ and the ratio of the continuum to $3.3 \mu \mathrm{m}$ band emission do not vary with distance from the central star (Sellgren 1989). Sellgren (1984) proposed that the emission arises from very small grains heated briefly to high temperature by absorbing a single ultraviolet photon.

The IRAS satellite detected 12,25 , and $60 \mu \mathrm{m}$ flux levels for highlatitude galactic cirrus in excess of the fluxes expected from the $100 \mu \mathrm{m}$ flux for grains in thermodynamic equilibrium with the interstellar radiation field (Boulanger \& Perault 1988). The excess fluxes can be explained by transient heating of very small grains, indicating that this population is widespread in the ISM. Boulanger et al. (1988) conclude that the very small grains are destroyed by very strong UV radiation, based on the low IRAS $12 \mu \mathrm{m}$ flux near some hot stars and in many Seyfert galaxies.

The population of grains causing the visual and near-infrared extinction and polarization is not so easy to characterize. They must be elongated and aligned to account for the observed polarization. The linear and circular polarization are best fit by a material such as silicate that is not 
strongly absorbing. Absorbing grains must also be present, because silicates alone would give too high an albedo. Mathis et al. (MRN 1977) pictured the silicate and carbon grains as bare and homogeneous, with a size distribution $n(a) \sim a^{-3.5}$ and an upper cutoff radius of $0.25 \mu \mathrm{m}$. Draine $\&$ Lee (1984) further developed the model of separate graphite and silicate components, deriving optical constants that fit the extinction curve from the UV through the infrared with the MRN size distribution. Their model requires fairly large graphite grains and there is no ready explanation how these would be formed. Considering the many processes an interstellar grain is subjected to, it is difficult to see how the carbon and silicate components remain separated.

Greenberg (1982, 1989 and refs. therein), recognizing that a typical IS grain cycles many times between molecular clouds and the diffuse ISM, developed a model of core-mantle grains. Within molecular clouds, icy mantles composed of the abundant elements $\mathrm{H}, \mathrm{C}, \mathrm{N}, \mathrm{O}$ accrete on existing silicate cores. When the grains are subsequently exposed to UV radiation, the mantles are photoprocessed, leaving an organic refractory residue. The optical properties of the residue depend on the $\mathrm{C} / \mathrm{H}$ ratio. In the limit of maximum processing, $\mathrm{C} / \mathrm{H}>1$ and the mantles would resemble amorphous carbon. When the grain cycles back into a molecular cloud, another layer of ices is deposited, and the process repeats. Thus, grains in the diffuse ISM have mantles of differing thickness and differing stages of photoprocessing.

Mathis \& Whiffen (1989) proposed that IS grains are fluffy conglomerates of small silicate, amorphous carbon, and graphite particles. They argue that grain growth in dense clouds takes place mainly by agglomeration, based on observations that small grains are deficient in the outer parts of molecular clouds. No doubt, both accretion and agglomeration take place in dense clouds, but until water ice begins to coat the grains enough to show a $3.07 \mu \mathrm{m}$ absorption feature, there is little volume available in the materials condensible from the gas. A major issue is what type of grain structure survives best in interstellar shocks. Do the photoprocessed mantles protect the silicate cores, as Greenberg proposes? Does the fluffy structure protect the inner parts of conglomerate grains as Mathis \& Whiffen propose?

Rowan-Robinson (1992) has proposed that there is an additional component of large $(\sim 30 \mu \mathrm{m})$ grains to account for the high millimeter 
emissivity of the dust in diffuse galactic emission and star-forming regions, where the dust absorption efficiency, $Q_{a b s} \propto \lambda^{-1}$, instead of $\propto \lambda^{-2}$ expected for small crystalline grains (Page et al. 1990). However, there is considerable evidence that amorphous materials can have a far-infrared wavelength dependence of $\mathrm{Q}_{\mathrm{abs}}$ less steep than $\lambda^{-2}$. Laboratory measurements of carbon by Koike et al. (1980) show a decrease closer to $\lambda^{-1}$. Huffman (1987) explains these results as due to the effects of particle clustering, a conclusion supported by Wright's (1987) calculations for fractal particles. Recently, Agladze et al. (1994) have measured the mass absorption coefficient, $\mathrm{x}$, of amorphous and crystalline silicate powders at millimeter wavelengths. They find that $\kappa \sim \lambda^{-1.3}$ for the amorphous powder and that $\kappa$ is $\sim 3$ times higher for the amorphous powder.

Any model of IS grains has to be consistent with cosmic elemental abundances. Assuming that cosmic abundances are the same throughout the ISM, we can infer the dust composition by observing the extent to which gas phase abundances are depleted relative to cosmic abundances. Because many atomic resonance lines are in the ultraviolet, UV spectroscopy is the most important means for studying gas phase depletions. In general, the depletions correlate with condensation temperature, although other factors, such as chemical reactivity, may also be important (Jenkins 1989). The most refractory elements $\mathrm{Al}, \mathrm{Ca}$, and $\mathrm{Ti}$ are depleted by factors up to $\sim 1000$, while $\mathrm{Fe}, \mathrm{Cr}$, and $\mathrm{Ni}$ are depleted by a factor of $\sim 100$.

Spitzer (1985) noted that characteristic depletion levels are associated with each phase of the ISM: dense clouds, diffuse clouds, and the warm neutral intercloud medium. These trends are supported by the recent high spectral resolution study of the $\zeta$ Oph line of sight (Savage $e t$ al. 1992).

Determining the gas phase depletions of two of the most important elements, Si and C, has been difficult. The f-value for the Si II $180.8 \mathrm{~nm}$ line is uncertain by a factor of two, while the $133.4 \mathrm{~nm}$ resonance line of C II is usually saturated. Moreover, the cosmic abundances of C,N,O are uncertain. Solar abundances of C,N,O differ by a factor of 2.5 to 1.5 from those in the atmospheres of young B stars, presumably typical of the ISM in the present epoch. This translates into a large uncertainty (factor $\sim 3$ for $\mathrm{O}, \sim 20$ for $\mathrm{C}$ ) in the abundance of these elements residing in dust (Sofia $e t$ al. 1994). 
Sofia et al. (1994) have reexamined the gas phase depletions for the major elements using new data from the Hubble Space Telescope ultraviolet spectrograph and improved f-values for Si II and Mg II. Among the major elements, Fe has the largest fraction of its atoms locked up in dust ( $283 \%$ ), while $z 34 \%$ of $\mathrm{Si}$ is in the dust phase. Oxygen is the most abundant element in the dust by number (because of its high cosmic abundance), greater than the sum of all other atoms. The ratios of $\mathrm{Fe}: \mathrm{Si}$ and $\mathrm{Mg}: \mathrm{Si}$ range from $1: 1$ to more than $2: 1$. The sum $(\mathrm{Fe}+\mathrm{Mg}): \mathrm{Si}$ is, in many cases, higher than can be accounted for by silicates of olivine $(\mathrm{Mg}, \mathrm{Fe})_{2} \mathrm{SiO}_{4}$ or pyroxene $(\mathrm{Mg}, \mathrm{Fe}) \mathrm{SiO}_{3}$. Thus, some of the $\mathrm{Mg}$ and $\mathrm{Fe}$ must form oxides or metallic Fe. The dust phase abundance of carbon is problematic. If one adopts the lower B star abundances, which seem to fit better for $\mathrm{O}$ and $\mathrm{N}, \mathrm{C}$ is only marginally depleted in the gas phase, scarcely sufficient to form the graphite grains required to produce the $0.2175 \mu \mathrm{m}$ bump, let alone the amorphous carbon and PAH material. The higher solar $\mathrm{C}$ abundance, while compatible with grain models, has to be viewed as an upper limit. Sulphur shows little or no gas phase depletion. Yet, FeS grains are common in interplanetary dust particles of possible IS origin.

\subsection{Evolution of Dust Grains in the Diffuse Interstellar Medium}

Grains condense in the circumstellar environment around masslosing AGB stars. Silicates form around M stars, where O $>C$; spectra of these stars show strong $10 \mu \mathrm{m}$ silicate features. Carbon and $\mathrm{SiC}$ condense around carbon stars, where $\mathrm{C}>\mathrm{O}$; the spectral feature of $\mathrm{SiC}$ is seen in these sources. The carbon grains are apparently amorphous, rather than graphite. The $0.2175 \mu \mathrm{m}$ feature is not seen in the spectra of $\mathrm{C}$ stars, but a broad feature near $0.24 \mu \mathrm{m}$ characteristic of amorphous carbon is occasionally present. The slope of the far infrared continuum also implies that the grains are amorphous. Wolf-Rayet stars, planetary nebulae, and supernovae are minor sources of dust. Based on the mass loss rates from AGB stars, the time scale for replenishing the dust in the ISM is a few times $10^{9}$ years.

But the diffuse ISM is a harsh environment for a tiny grain. There are strong theoretical arguments that fast shocks associated with supernovae remnants efficiently destroy grains by thermal and non-thermal 
sputtering and collisions (Seab \& Shull 1986; McKee 1989; Jones et al. 1994). Thermal sputtering destroys small grains, while the refractory cores of larger grains may be protected by mantles. Grain-grain collisions annihilate grains and shatter grains, leaving the fragments vulnerable to sputtering; even fairly large grains can be destroyed. There is ample evidence for grain destruction from the gas phase abundances in high velocity clouds; some clouds show almost no depletion of the major grain-forming elements $\mathrm{Fe}$ and $\mathrm{Si}$. The efficiency of grain destruction and the effective supernova rate near the galactic plane are somewhat uncertain. McKee (1989) and Jones et al. (1994) derive a destruction lifetime of $\sim 4$ $\mathrm{x} 10^{8} \mathrm{yrs}$, far shorter than the timescale for replenishment from AGB stars.

Thus, not only are grains heavily processed in the ISM, but also significant grain growth must occur in the ISM. McKee (1989) points out that the bulk of elemental silicon and other refractory elements (except carbon) enter the ISM in the gas phase from supernovae, supporting the conclusion that much of the grain growth has to take place in the ISM. Most of the grain destruction takes place in the warm neutral phase of the ISM. Since grain growth occurs primarily in dense regions, there must be rapid exchange between molecular clouds and the warm neutral medium.

Observational evidence for grain destruction and growth comes from tracing the patterns of gas phase depletion. The high resolution ultraviolet spectrograph on HST has opened a new window by allowing one to obtain depletion patterns for individual clouds of different velocity along a line of sight. The depletion patterns can give us insight into the grain chemistry. For example, Sofia et al. (1993) found that most Si is in the gas phase in a high-velocity cloud towards $\mu \mathrm{Col}$, while $\mathrm{Fe}$ remains depleted, implying that not all the $\mathrm{Fe}$ is contained in silicates. We can expect interesting new results on the details of grain chemistry to emerge as more lines of sight are surveyed by HST.

The SiC emission feature at $11.15 \mu \mathrm{m}$ is commonly seen in spectra of carbon stars. Yet, no corresponding SiC feature is seen in spectra of the ISM, setting an upper limit of $\sim 5 \%$ to the amount of Si tied up in $\mathrm{SiC}$ relative to that in silicates (Whittet et al. 1990). To explain the lack of SiC in the ISM and the rarity of IS SiC grains in primitive meteorites, Whittet et al. conclude that $\mathrm{SiC}$ grains are destroyed by oxidation in diffuse IS clouds on time scales of $\sim 5 \times 10^{7}$ yrs.

Clearly, as observations from the UV to the infrared expand, our 
model of IS grains has to become more sophisticated. Some of the present questions are:

1. How much carbon is present in the dust? Is it sufficient to cover the graphite, PAH, and amorphous carbon in the grain models? What is the most common form of carbon?

2. Is the $0.2175 \mu \mathrm{m}$ extinction bump due to graphite? Why is $\lambda_{\max }$ constant? Where does the graphite form?

3. How do very small grains survive without being destroyed or incorporated into larger grains?

4. How does a significant component of oxides affect the "working hypothesis" model of grain populations?

5. What is the size distribution and physical structure of the "classical" grains responsible for the visual extinction?

6. How do grains survive shocks?

\section{DUST IN THE SOLAR SYSTEM}

\subsection{Cometary Dust}

Comets formed in the outer solar nebula, where the temperature remained low enough that intact interstellar grains could have been incorporated into the comet nuclei. Except for alteration of the outer few meters by cosmic rays over the lifetime of the solar system, the material was not processed further until the comet approached the sun in the current epoch.

Comets apparently originate from two distinct reservoirs in the outer solar system. Short-period comets, with their low-inclination orbits, are thought to originate in the Kuiper Belt at $\sim 30-100$ AU (Duncan, Quinn, \& Tremaine 1988). They typically have low to moderate activity, from limited active areas on the nucleus. The comets that populate the inner and outer Oort Cloud probably formed in the Saturn - Neptune region (10-30 AU) and were dynamically scattered to the Oort Cloud. The Oort Cloud is the source of new and long-period comets.

Time-of-flight mass spectrometers on 3 Halley space probes measured the elemental composition of submicron sized dust particles in the coma (Kissel et al. 1986). A dust component rich in refractory organic 
material was discovered, the so-called CHON particles. Elemental abundances of the major rock-forming elements are approximately chondritic (Jessberger et al. 1988). Carbon is enriched by a factor of $\sim 10$; in this sense, cometary material is more "primitive" than carbonaceous chondrite meteorites. In most cases, $\mathrm{C}, \mathrm{Si}$, and $\mathrm{Mg}$ occur together, indicating that the silicate and carbonaceous material are well mixed on a submicron scale, similar to chondritic aggregate IDPs (section 4.2). But the total mass of material measured during the Halley flybys is less than one $10 \mu \mathrm{m}$ IDP!

Spectra of Halley in the infrared and millimeter regions indicated that a variety of organic molecules were present. Chief among the presumed solid state features was a broad unidentified feature at $3.36 \mu \mathrm{m}$, subsequently seen in a number of comets. Reuter (1992) has shown that up to half of the flux in the $3.36 \mu \mathrm{m}$ feature may be due to methanol, leaving a mystery as to what constitutes the remainder of the feature. The carrier could be either an organic molecule or small organic refractory grains. The feature does not match spectral features seen in any IS or circumstellar source, although there is admittedly considerable uncertainty in the shape of the residual cometary feature. The spatial distribution of carbon-bearing molecules in the coma of Halley implied a distributed source in addition to the nucleus, indirect evidence for a volatile organic species.

In the interstellar medium, a series of emission bands are seen in carbon-rich sources at $3.29,6.2,7.7,8.6$, and $11.3 \mu \mathrm{m}$. These are generally attributed to vibrations in polycyclic aromatic hydrocarbons, although exact spectral matches have been difficult to pinpoint. This series of bands has not yet been detected in a comet. If comet material is composed of interstellar material, this result is surprising. Airborne 5-8 $\mu \mathrm{m}$ spectra exist only for comets Halley and Wilson; the 6.2 and $7.7 \mu \mathrm{m}$ features were not detected. However, a peak at $3.28 \mu \mathrm{m}$ which may be related to the interstellar PAH feature is sometimes present shortward of the $3.36 \mu \mathrm{m}$ band in comets. The $3.28 \mu \mathrm{m}$ peak was particularly pronounced in longperiod Comet Levy 1990 XX (Davies et al. 1991); thus, the carrier of the $3.28 \mu \mathrm{m}$ feature may vary in abundance among comets.

Many new and long period comets display silicate emission in filter photometry. Of the few comets with $8-13 \mu \mathrm{m}$ spectra, four (long-period comets Bradfield 1987 XXIX and Levy 1990 XX, new comet Mueller 
1993a, and P/Halley) exhibit a strong silicate feature with a distinct peak at $11.2 \mu \mathrm{m}$, attributed to crystalline olivine particles, in addition to a broader maximum near $9.7 \mu \mathrm{m}$ (Hanner et al. 1994a,b). The olivine could have formed at high temperatures in the inner solar nebula, in which case there must have been considerable radial mixing in the solar nebula to transport these grains to the region of comet formation. Or, the grains may be presolar, in which case it is puzzling that the $11.2 \mu \mathrm{m}$ peak is rarely seen in interstellar dust:

But of the 5 new comets with spectra, only Mueller 1993a shows strong silicate emission with a $11.2 \mu \mathrm{m}$ peak. Each of the others has a unique, and puzzling, $10 \mu \mathrm{m}$ spectrum. For example, Wilson $1987 \mathrm{VII}$ had a broad amorphous feature, perhaps a consequence of cosmic ray processing of the outer layers in the Oort Cloud. No short-period comet except P/Halley has shown a silicate feature. (Halley may well be a captured long period comet.) Some new comets also lack a silicate feature. Is the composition of these comets really different or is there simply a lack of the small particles needed to produce a feature?

The polarization phase curve, $\mathrm{P}(\theta)$, has been measured for a number of comets (Dollfus et al. 1988; Levasseur-Regourd \& Hadamcik 1994 and refs. therein). All of the comets have negative polarization at small phase angles, a neutral point at $\theta_{0} \sim 21^{\circ}$, slope $h \sim 0.20-0.25 \%$ per deg at $\theta_{0}$, and maximum polarization near $90^{\circ}$ phase. However, they separate into two distinct groups having $\mathrm{P}_{\max } \sim 10 \%$ or $\sim 25 \%$. The higher $\mathrm{P}_{\max }$ correlates with comets that display a strong silicate feature, while the comets with lower $\mathrm{P}_{\max }$ have weak or no silicate emission. High $\mathrm{P}_{\max }$ and strong silicate emission are characteristics of small grains, $a<1 \mu \mathrm{m}$. Thus, the two groups of comets could be distinguished mainly by differences in their size distribution, although compositional differences are also possible. Spatially resolved observations of the inner coma show increased polarization in dust jets and a decrease in the near-nucleus halo. These observations could indicate that the optical properties of the dust change after release from the nucleus, possibly due to sublimation of ices.

Thus, cometary dust is not the same in all comets nor even in one comet at all times. The infrared spectra demonstrate that comet dust is not $100 \%$ interstellar dust. At least some of the interstellar silicate material must have been reprocessed to produce the $11.2 \mu \mathrm{m}$ spectral peak. Comets are rich in organic material, both molecules and carbon-rich grains, but the 
material is not yet well characterized. Fundamental questions remain about how comets fit into the evolutionary picture. Some of these questions can only be answered from direct sampling of cometary dust:

1. What is the heterogeneity among comets and within one comet nucleus?

2. Can the differences among comets in optical properties such as polarization be explained solely by differences in particle size or are compositional differences required?

3. Is the material in comets "local"; i.e., local to the solar nebula at a given heliocentric distance, or is it a mixture of material from high and low temperature regions of the nebula?

4. To what extent are intact IS grains preserved in comets?

\subsection{Interplanetary Dust}

The earth is situated within a flattened cloud of interplanetary dust. To first order, the cloud is symmetric about a plane inclined $1.5^{\circ}-3.0^{\circ}$ to the ecliptic. The particle size distribution extends from submicron grains up to sizes larger than a centimeter and, indeed, up to kilometer sized comets and asteroids. We refer to the particles $<100 \mu \mathrm{m}$ in size as dust. Since the dynamical lifetime of the dust is short compared to the age of the solar system, the dust cloud must be continually replenished.

Comets and asteroids are the main sources for replenishing the dust cloud. Dust bands and dust trails seen in the IRAS sky flux maps provide evidence of these sources. The dust bands are associated with asteroid families and represent debris from collisional breakup of these families. The dust trails extend for several degrees along the orbits of comets; they consist of millimeter sized particles (Sykes \& Walker 1992). The size distribution of the interplanetary dust evolves with time due to collisions as the particles slowly spiral inward towards the sun due to Pointing-Robinson drag.

Interplanetary dust can be studied several ways: remote sensing of the scattered solar radiation and thermal emission from the dust; in situ detection of dust impacts, leading to the mass distribution and orbital parameters; and laboratory analysis (structure, mineralogy) of samples collected at Earth. 
The zodiacal light brightness viewed from earth is an integral over the scattering by all dust particles along the line of sight. That is, the spatial distribution, size distribution, and optical properties of the dust are convolved into one integral. The size distribution and optical properties may vary along the line of sight due to dust particle collisions, heating, and mixing of different populations with different orbital distributions. The intensity and polarization at visual wavelengths are measured as a function of ecliptic latitude and angular distance from the sun. Thermal emission from the interplanetary dust has been measured from rocket flights and from the IRAS and COBE satellites. At 5-50 $\mu \mathrm{m}$, the emission is the strongest component of the diffuse sky brightness beyond the terrestrial atmosphere.

Inversion techniques have been applied to extract the local properties of the dust from the line-of-sight integral (Levasseur-Regourd et al. 1990). The polarization at phase angle $\theta=90^{\circ}$ is $\sim 30 \%$ at $1 \mathrm{AU}$ in the symmetry plane, decreasing with decreasing radial distance, $r$, and decreasing with increasing distance, $z$, above the symmetry plane. The $12 \mu \mathrm{m} / 25 \mu \mathrm{m}$ color temperature is found to vary as $\mathrm{T}_{0} r^{-n}$, where $n=0.35 \pm 0.03$, in contrast to $n=0.5$ expected for a blackbody. The color temperature at $1 \mathrm{AU}, \mathrm{T}_{0}$, is sensitive to the absolute calibration of the infrared data; it lies in the range $260-295 \mathrm{~K}$ in the symmetry plane and decreases with increasing $z$. The albedo $A_{p}(\theta)$ is $0.08-0.14$ at $1 \mathrm{AU}$ and $\theta=90^{\circ}$, with a radial gradient $r^{-0.3 \pm 0.1}$. The albedo increases with $z$. Thus, higher albedo is associated with lower polarization and lower temperature relative to a blackbody. The trends with $z$ imply the existence of at least two distinct dust populations with differing optical properties and orbital inclinations. The trends with $r$ in the symmetry plane could be explained either by two or more distinct dust populations with different $r$-dependence or by the evolution of size and optical properties as the particles spiral in towards the sun.

Dust detectors have flown on a number of spacecraft in earth orbit and solar orbit. The mass, speed, and direction of the impacting particle can be measured (Grün et al. 1985). The mass density at $1 \mathrm{AU}$ in the ecliptic is $\sim 10^{-19} \mathrm{~kg} / \mathrm{m}^{3}$. The mass function peaks at $10^{-4}-10^{-6} \mathrm{~g}$, while the cross-sectional area (source of the zodiacal light) peaks at $\sim 10^{-6}-10^{-7} \mathrm{~g}$ at $1 \mathrm{AU}$ in the ecliptic. There is evidence for different dust populations 
with different orbital characteristics, confirming the inferences from remote sensing (Grün et al. 1985; Divine 1993).

The dust impact experiment on the Ulysses spacecraft has detected interstellar dust particles at $5 \mathrm{AU}$ that are streaming through the solar system from the apex direction of solar motion (Grün et al. 1994). The dust velocities of $26 \pm 4 \mathrm{~km} / \mathrm{s}$ agree with the velocity of interstellar Helium gas entering the solar system. The mean mass of the particles, $3 \times 10^{-13} \mathrm{~g}$, is larger than the "classical" $0.1 \mu \mathrm{m}$ IS grains, but consistent with a model of aggregate IS particles. Smaller IS grains may be prevented from approaching within $5 \mathrm{AU}$ of the sun by the Lorentz force. This discovery raises the interesting possibility of directly collecting a sample of interstellar dust for laboratory analysis.

Interplanetary dust particles (IDPs) in the size range $5-50 \mu \mathrm{m}$ are routinely collected in the stratosphere, having survived atmospheric entry without destructive heating (Bradley et al. 1988). They fall into three compositional groups, chondritic ( $\sim 60 \%)$, iron-sulphur-nickel $(\sim 30 \%)$, and mafic silicate $(\sim 10 \%)$, often appearing together in one IDP. The chondritic aggregate IDPs are fine-grained aggregates of micrometer-submicrometer sized particles of silicate and carbonaceous material, with chondritic abundances of the major rock-forming elements and high carbon content. They can be divided into olivine, pyroxene, and layer lattice silicate classes, based on the dominant silicate mineral. The olivine-rich and pyroxene-rich particles have a porous structure, while the layer lattice silicates have a smoother texture. The fine-grained aggregate structure is distinctly different from meteoritic material, even "primitive" carbonaceous chondrites. Pyroxene whiskers and platelets that bear the signatures of direct vapor phase condensation have been found. Their unusual shapes and growth patterns point to growth from the vapor phase at low pressures.

Cometary particles are more likely to contain IS grains than asteroidal debris. But how does one identify cometary IDPs? The high carbon content, fluffy structure, and wide range in $\mathrm{Mg} / \mathrm{Fe} / \mathrm{Si}$ make the pyroxene-rich IDPs the best candidates. One means of distinguishing their origin is to look for evidence of heating. Cometary particles on elliptical orbits will have higher entry velocities than asteroidal particles and consequently will experience stronger atmospheric heating. Hydrated silicate particles apparently suffered only minor heating, consistent with an asteroidal origin, while the olivine IDPs were, on average, strongly heated. 
Pyroxene-rich particles are intermediate. There may be a strong selection effect against cometary particles with fragile structure or with components that are volatile at temperatures below $600^{\circ} \mathrm{C}$.

No single class of IDPs produces a silicate emission feature resembling cometary silicate spectra (Sandford \& Walker 1985). The spectra of olivine IDPs match the $11.25 \mu \mathrm{m}$ cometary peak, but not the overall shape. While a mixture of all three IDP silicate classes approximately matches the cometary feature, one is then left with a puzzle why the three classes are so distinct. Bradley et al. (1992) discovered two very porous aggregates whose spectra are qualitatively similar to the cometary spectra. These IDPs contain abundant glass and tiny $(0.1 \mu \mathrm{m})$ pyroxene crystals. A few larger olivine crystals give rise to the $11.25 \mu \mathrm{m}$ peak. But, if cometary silicates are predominantly glassy, in order to explain the $9.8 \mu \mathrm{m}$ spectral feature, why are glassy IDPs so rare? In summary, while the pyroxene-rich IDPs are the most likely cometary particles, many inconsistencies remain to be explained.

The most striking feature of the porous aggregate IDPs is that they are unequilibrated mixtures of high and low temperature condensates even on a submicrometer scale. Does this reflect efficient mixing of small grains formed in different regions of the solar nebula and transported to the region of comet formation? Or, are these submicrometer units truly interstellar grains? A major component of these IDPs are compact grains 0.1-0.5 $\mu \mathrm{m}$ in size, given the descriptive name of GEMS (Glass with Embedded Metal and Sulfides). Bradley (1994) argues that the GEMS show evidence of high radiation doses (such as eroded rims and $\mathrm{Mg}, \mathrm{Si}$ depletion relative to O) that could have occurred only in the ISM. Contrary to earlier description of the GEMS as "tar balls", they do not contain carbon; rather, their dark appearance is due to $\mathrm{Ni}-\mathrm{Fe}$ beads dispersed in the silicate glass. Formation of these metallic beads is a consequence of irradiation of silicates. Are the GEMS truly interstellar "dirty silicate" grains?

If indeed the GEMS are interstellar, we have the exciting prospect of studying in the laboratory the physical processes to which IS grains have been subjected. 


\subsection{Interstellar Material in Meteorites}

Meteorites are an abundant source of extraterrestrial material that may contain IS components. Since meteoritic material has been subjected to considerable solar system processing, isotopic anomalies are the only sure tracers of interstellar grains; these identifications rely not on one isotopic ratio, but a pattern. Most of the analysis has been focused on carbonaceous chondrites, the most "primitive" meteorite class, particularly the C2 meteorites Murchison and Murray. Four types of IS grains have been identified, based on non-solar isotope ratios in $\mathrm{C}, \mathrm{N}, \mathrm{O}, \mathrm{Mg}, \mathrm{Si}, \mathrm{Ti}$, and the noble gases $\mathrm{Ne}, \mathrm{Kr}$, Xe (Anders \& Zinner 1993).

Diamonds: Microdiamonds $0.001 \mu \mathrm{m}$ in size are the most abundant IS component yet isolated, comprising $\sim 400 \mathrm{ppm}$ of the meteoritic material. They contain xenon enriched in both the light $\left({ }^{124} \mathrm{Xe},{ }^{126} \mathrm{Xe}\right)$ and heavy $\left({ }^{134} \mathrm{Xe},{ }^{136} \mathrm{Xe}\right)$ isotopes, indicative of both p-process and r-process nucleosynthesis occurring in supernovae. Although the xenon points to origin in supernovae, the formation process and the process for trapping xenon are not clear. Allamandola et al. $(1992,1993)$ have detected an infrared absorption feature at $2880 \mathrm{~cm}^{-1}$ that they attribute to a tertiary $\mathrm{CH}$ group in microdiamonds. The derived abundance in four dense clouds gives a lower limit of $5 \%$ of the cosmic $\mathrm{C}$ abundance tied up in these microdiamonds. The spectral feature is not seen in the diffuse ISM, although it may be masked by aliphatic spectral features. If microdiamonds are widespread in the ISM, then a supernova origin for the microdiamonds may be insufficient.

$\mathrm{SiC}$ : SiC grains, have been found at the $7 \mathrm{ppm}$ level. Their size ranges from $0.3-3 \mu \mathrm{m}$, although a few as large as $10 \mu \mathrm{m}$ are seen. The xenon isotopes ${ }^{128} \mathrm{Xe}-{ }^{132} \mathrm{Xe}$ follow a pattern indicative of s-process nucleosynthesis in AGB stars. $\mathrm{Kr}$ isotopes set limits on the temperature and s-process time scale that are consistent with calculations for $1-3 \mathrm{M}_{0}$ thermally pulsing AGB stars. The ${ }^{12} \mathrm{C} /{ }^{13} \mathrm{C}$ ratio of most grains is consistent with that observed in carbon stars. Differences in isotopic ratios among $\mathrm{SiC}$ grains, specifically ${ }^{30} \mathrm{Si} /{ }^{28} \mathrm{Si}$ and ${ }^{29} \mathrm{Si} /{ }^{28} \mathrm{Si}$, can only be explained by nucleosynthesis in different stars with different metallicities. The range of oxygen isotopic abundances measured in certain oxide grains also seems to require origin in stars of differing mass and initial ${ }^{16} \mathrm{O} /{ }^{18} \mathrm{O}$. An excess 
of ${ }^{21} \mathrm{Ne}$ in some grains, most likely a result of cosmic ray spallation, implies a presolar exposure age up to $10^{9}$ years.

Graphite: Several forms of graphite have been identified in primitive meteorites at the $2 \mathrm{ppm}$ level, but only the spherical grains 0.8 $7 \mu \mathrm{m}$ in size have anomalous $\mathrm{C}$ isotopes proving their IS origin. These spheres are fairly well ordered crystalline graphite with concentric structure. The graphite has a wide range in ${ }^{12} \mathrm{C} /{ }^{13} \mathrm{C}$, including ratios up to .7200 . Excess ${ }^{26} \mathrm{Mg}$, due to decay of ${ }^{26} \mathrm{Al}$, allows one to infer the original ${ }^{26} \mathrm{Al} /{ }^{27} \mathrm{Al}$ abundance. These IS graphite grains have ${ }^{26} \mathrm{Al} /{ }^{27} \mathrm{Al}$ ranging from $10^{-4}-10^{-1}$, compared to the solar system ratio of $5 \times 10^{-5}$. Graphite is presumed to be common in the ISM as the source of the $0.22 \mu \mathrm{m}$ extinction bump. However, the IS graphite particles are $\sim 0.01 \mu \mathrm{m}$, in contrast to the large graphite grains in the meteorites.

Corundum $\left(\mathrm{Al}_{2} \mathrm{O}_{3}\right)$ : Corundum grains show a large range in their oxygen isotopes and inferred ${ }^{26} \mathrm{Al} /{ }^{27} \mathrm{Al}$ ratios range up to $2 \times 10^{-2}$. The range in oxygen isotopic ratios seem to require origin in stars of differing mass and initial ${ }^{16} \mathrm{O} /{ }^{18} \mathrm{O}$.

In addition to these grains, another component of IS material exists in meteorites, associated with anomalous deuterium/hydrogen ratios (Zinner 1988). In the diffuse ISM, the relative $\mathrm{D} / \mathrm{H}$ abundance is $\sim 10^{-5}$. Some molecules in molecular clouds are observed to have $\mathrm{D}$ enrichments $10^{2}-10^{4}$ above this value, generally attributed to low temperature ionmolecule reactions. The estimated $\mathrm{D} / \mathrm{H}$ in the protosolar nebula is $\sim 2 \times 10^{-5}$. Terrestrial standard mean ocean water (SMOW) has $\mathrm{D} / \mathrm{H}=1.57 \times 10^{-4}$, a factor of 8 times the protosolar value. The $\mathrm{D} / \mathrm{H}$ in water molecules measured by the neutral mass spectrometer on the Giotto Halley probe was $0.6 \times 10^{-4} \leq \mathrm{D} / \mathrm{H} \leq 4.8 \times 10^{-4}$. This ratio is compatible with other solar system hydrogen that was accreted as part of volatile molecules (Titan, Earth), in contrast to the gaseous hydrogen in the solar nebula (Eberhard et al. 1987).

$\mathrm{D} / \mathrm{H}$ anomalies up to a factor of 6 times SMOW (50 times protosolar nebula $\mathrm{D} / \mathrm{H}$ ) have been measured in a variety of meteorites. The anomalies have been traced to acid-insoluable organic residues, constituting only a small fraction of the meteoritic material. The D enrichment is not correlated with other isotopic anomalies, such as ${ }^{13} \mathrm{C} /{ }^{12} \mathrm{C}$. There may also be an acid-soluble organic component with high $\mathrm{D} / \mathrm{H}$. The presence of a D-enriched organic component is evidence that at least some 
presence of a D-enriched organic component is evidence that at least some meteoritic materials escaped strong heating in the solar nebula.

$\mathrm{D} / \mathrm{H}$ anomalies up to a factor of 3.5 times SMOW have been found in IDPs (McKeegan et al. 1985). The $\mathrm{D} / \mathrm{H}$ ratio has a large range within a single IDP. Ion probe images show that the deuterium is confined to a small volume of the IDP and is associated with carbon. Deuterium enrichment is seen in about half of the IDPs analyzed, including both pyroxene-rich anhydrous particles and the layer lattice silicate class. The enriched material constitutes a larger fraction of the IDPs than in the case of meteorites.

The meteoritic evidence leads to two important conclusions. First, the solid grains in the protosolar nebula came from a variety of steller sources, including supernovae and AGB stars with a range of metallicities. Second, some grains did survive the harsh IS environment and retained the imprint of their formation. We are also left with a number of puzzles.

Some of the unanswered questions are:

1. Are there other IS grains in meteorites with normal isotopic abundances? How could such grains be identified?

2. If graphite is the producer of the $0.22 \mu \mathrm{m}$ bump in the IS extinction, why is it so rare in meteorites and IDPs?

3. What is the origin of the microdiamonds? Do they cause the spectral feature at $2880 \mathrm{~cm}^{-1}$ in dense IS clouds?

4. Why are the $\mathrm{SiC}$ and graphite grains so much larger than the canonical IS grains?

\section{EVOLUTION OF DUST FROM THE INTERSTELLAR MEDIUM TO THE SOLAR NEBULA}

\subsection{Evolution of Dust in Dense Regions}

The optical and physical properties of grains do not remain static over time. Grain growth and destruction, structural transformations, and chemical evolution all affect grain properties. The rate of grain destruction in the diffuse IS medium implies that considerable grain growth must take place in dense clouds. Gas phase depletions are generally larger in dense regions, indicating growth of solid grains. 
Numerous observational studies of the extinction and polarization have shown that the mean grain size is larger within dense clouds. Grain size is related to two observational parameters. The ratio of total to selective extinction, $R=A_{V} / E(B-V)$, increases with grain size. In the diffuse ISM, $\mathrm{R}=3.1$, whereas the mean $\mathrm{R}$ is 4.0 in the $\rho$ Oph dark cloud (Vrba et al. 1993) and $z 4.5$ in the R CrA cloud (Vrba et al. 1981). The IS polarization $v s \lambda$ has a characteristic shape, when plotted as $\mathrm{P} / \mathrm{P}_{\max } v s$ $\lambda_{\max } / \lambda$. The wavelength of maximum polarization, $\lambda_{\max }$, shifts towards longer $\lambda$ as the grain size increases. Vrba et al. (1993) find a mean value of $\lambda_{\max }=0.645 \mu \mathrm{m}$ in the $\rho$ Oph dark cloud, compared to $\lambda_{\text {max }}=0.545 \mu \mathrm{m}$ in the diffuse ISM. In the R CrA cloud, $\lambda_{\max }=0.753 \mu \mathrm{m}$. The R CrA cloud is a young region, in which star formation is not far advanced. Thus, large grains have formed at an early evolutionary stage in this cloud.

The $0.2175 \mu \mathrm{m}$ extinction feature is generally broader in dense clouds than in the diffuse ISM. The growth of amorphous mantles on small graphite grains is a likely explanation for the broader feature (Mathis 1994). Sorrell (1990) proposes that the bump is broadened by accretion of $\mathrm{H}$ atoms on the surface of graphite grains. Hydrogenation of carbon can also explain the decrease in band strength that is associated with larger $\mathrm{R}$ in some regions.

Grain growth can occur by accretion or coagulation. The higher gas depletions and the presence of the $3.08 \mu \mathrm{m}$ water ice absorption feature (and other spectral features of ices; section 5.2) indicate that accretion of icy mantles has occurred in dense clouds. Grain growth by accretion will lead to higher extinction relative to the hydrogen column density, $\mathrm{N}_{\mathrm{H}}$. Yet, some lines of sight have higher $\mathrm{R}$ and lower than usual $\mathrm{A}_{\mathrm{V}} / \mathrm{N}_{\mathrm{H}}$. Moreover, some lines of sight yield higher $\mathrm{R}$ and $\lambda_{\max }$ but lack a $3.08 \mu \mathrm{m}$ ice band. If the sticking coefficient is independent of grain radius, then accretion will increase all radii by an equal amount. The larger grains in the size distribution will then increase in size by only $10 \%-20 \%$, not sufficient to account for the anomalous $\mathrm{R}$ and $\lambda_{\max }$ in some clouds.

Thus, grain growth by coagulation must also be important in dense regions. Coagulation requires low velocity collisions. Sticking of bare grains occurs only at velocity $\leqslant 1 \mathrm{~m} / \mathrm{s}$ (Tielens 1989). Sticking is successful at somewhat higher velocities if the grains are ice-coated. Thus, accretion enhances coagulation. 
We expect the dust in dense clouds, then, to have a variety of shapes, sizes, and compositions, including fluffy aggregates. The optical properties of such heterogeneous aggregates are difficult to specify. Two papers have recently presented working models for the dust optical properties in the dense regions from which protostars form. Preibisch et al. (1993) used three dust components: amorphous carbon, small silicate grains, and silicate cores with dirty ice mantles. They show that the presence of ice mantles can have a strong influence on the opacity of protostellar envelopes. Carbon grains embedded in icy mantles absorb more efficiently than the bare carbon grains of comparable size. Pollack et al. (1994) base their model, in part, on the chemical composition of primitive solar system materials. In contrast to other models, organic materials are the main reservoir for carbon. Metallic iron is a major absorbing species and troilite ( $\mathrm{FeS}$ ) is also explicitly included, along with glassy olivine and orthopyroxene.

Formation of the first protostars within a dense cloud can radically alter the surrounding dust, particularly if the protostars are massive, with a strong UV flux creating an H II region. This can result in evaporation of icy mantles and photolysis of grain mantles. Protostars develop strong winds, creating shock fronts that can lead to grain destruction. Within the protostellar core, coagulation and localized heating can occur.

Thus, dust grains evolve in complex ways within dense clouds. In the next section we examine the chemical changes that take place in grain mantles.

\subsection{Evolution of Ices and Organic Compounds}

There is ample evidence that icy mantles accrete on grains in cold dense regions. Gas atoms colliding with cold IS grains at temperature of $\sim 10 \mathrm{~K}$ will stick with essentially unit probability. Species such as $\mathrm{H}$ and $O$ will be mobile on grain surfaces and will react with other species to form new molecular compounds not necessarily present in the gas phase. The nature of these grain surface reactions depends on the availability of atomic $\mathrm{H}$ and $\mathrm{O}$. When the gas density is $\leqslant 10^{4} \mathrm{~cm}^{-3}$, atomic $\mathrm{H}$ is available to form reduced compounds such as $\mathrm{H}_{2} \mathrm{O}, \mathrm{H}_{2} \mathrm{CO}$, and $\mathrm{CH}_{3} \mathrm{OH}$. At high densities, where the hydrogen is bound up in $\mathrm{H}_{2}$, grain surface chemistry will depend on the abundance of atomic oxygen. 
Infrared spectroscopy of sources located within or behind dense clouds reveals many absorption features due to vibrational modes in ices and organic species. The prototypical source is the embedded protostar W33A. Laboratory investigations of the spectra of low-temperature ices and ice mixtures, before and after irradiation, has led to the identification of the major species, but important ambiguities remain (d'Hendecourt et al. 1986; Tielens \& Allamandola 1987; Grim et al. 1989).

The strong feature at $3.08 \mu \mathrm{m}$ due to the $\mathrm{OH}$ stretching vibration in $\mathrm{H}_{2} \mathrm{O}$ ice is seen in many embedded sources, consistent with water ice as the main constituent of grain mantles. The broad line profile is consistent with amorphous, rather than crystalline ice, as expected at low temperatures (Leger et al. 1983). The $2.97 \mu \mathrm{m}$ feature on the wing of the water band was previously ascribed to $\mathrm{NH}_{3}$, but is now thought to be due to scattering by relatively large ice grains (Knacke \& McCorkle 1987; Tielens 1989). The broad long-wavelength wing was also originally attributed to $\mathrm{NH}_{3}$, but is now thought to be $\mathrm{C}-\mathrm{H}$ vibrations in organic molecules. The $\mathrm{OH}$ bending mode absorption band at $6.0 \mu \mathrm{m}$ is seen in $\mathrm{W} 33 \mathrm{~A}$ and other sources.

$\mathrm{CO}$ is an abundant gas phase molecule. Solid $\mathrm{CO}$ has a band at $4.67 \mu \mathrm{m}$ and this feature has been detected in the spectra of a number of embedded protostars (Lacy et al. 1984; Geballe 1986; Tielens et al. 1991). The central wavelength, width, and profile of the solid CO band depend upon the ice mixture in which the $\mathrm{CO}$ is embedded, the temperature, and the amount of UV photolysis (Sandford et al. 1988). The astronomical feature usually has two components, a narrow absorption at $2140 \mathrm{~cm}^{-1}$, and a broader absorption centered at $2134 \mathrm{~cm}^{-1}$. Based on the laboratory spectra, the broader component arises from $\mathrm{CO}$ mixed at concentrations of a few percent in a polar ice, probably water ice. The narrower band at $2140 \mathrm{~cm}^{-1}$, however, must be due to $\mathrm{CO}$ in a non-polar ice, such as pure $\mathrm{CO}, \mathrm{CO}_{2}, \mathrm{~N}_{2}$, or $\mathrm{O}_{2}$ (Sandford et al. 1988; Tielens et al. 1991). Thus, regions with differing grain chemistry exist within a dense cloud. In a reducing environment, when gas phase hydrogen is in atomic form, $\mathrm{H}_{2} \mathrm{O}$ ice dominates the grain mantles, leading to the broader $\mathrm{CO}$ feature. When icy mantles accrete in an inert or oxidizing environment, in contrast, nonpolar ices form. Since the $\mathrm{H}_{2} / \mathrm{H}$ abundance depends on the gas density, the differences in grain mantle composition could reflect regions of differing gas density. Temperature could also be a factor, particularly in the 
environment around a protostar; the non-polar ices sublimate at a lower temperature than $\mathrm{H}_{2} \mathrm{O}$ ice. Palumbo \& Strazzula (1993) emphasized the importance of irradiation in broadening the $\mathrm{CO}$ feature. They conclude that irradiated samples provide a better fit to W33A and other published spectra.

The abundance of methanol $\left(\mathrm{CH}_{3} \mathrm{OH}\right)$ in grain mantles is controversial. Methanol could form via grain surface reactions when atomic $\mathrm{H}$ is available or as a product of UV photolysis or ion irradiation (although $\mathrm{H}_{2} \mathrm{CO}$ is the favored product). The $3.53 \mu \mathrm{m}$ absorption band in W33A has been identified as solid methanol by Grim et al. (1991). They derive a methanol abundance of $7 \%$ relative to water ice. However, Allamandola et al. (1992) compute an abundance of $40 \%$ relative to water ice for the $3.53 \mu \mathrm{m}$ band in W33A. Part of the problem lies in defining the baseline flux, since this feature lies in a spectral region of other broad shallow absorption. Moreover, a strong feature at $6.85 \mu \mathrm{m}$ in W33A may be due to methanol. To fit this feature in W33A requires a methanol abundance relative to water of $-50 \%$. Schutte et al. (1991) searched for the $\mathrm{C}-\mathrm{O}$ stretching band of methanol near $9.8 \mu \mathrm{m}$ in the spectra of 4 protostars (but not including W33A). From fits to the strong silicate absorption, they place a conservative upper limit of $6 \%-17 \%$ to the methanol/water ice abundance in these sources. Therefore, either the methanol abundance in grain mantles is very variable or the $6.85 \mu \mathrm{m}$ feature has a different origin. Skinner et al. (1992) detected the $9.8 \mu \mathrm{m}$ absorption towards the embedded source GL 2136, obtaining a methanol abundance of $~ 8 \%$ relative to water in the ice mantles. The intensity of the $6.85 \mu \mathrm{m}$ feature along this line of sight would require a methanol abundance almost 10 times higher. This seems to exclude an assignment to methanol for the $6.85 \mu \mathrm{m}$ feature. Possibly the $6.85 \mu \mathrm{m}$ band arises in more complex organic species, such as alcohols (Tielens \& Allamandola 1987). Laboratory experiments indicate that a $6.85 \mu \mathrm{m}$ feature is produced by irradiation and subsequent warmup of ice mixtures containing $\mathrm{NH}_{3}$ and $\mathrm{CO}$ (Grim et al. 1989).

Even with the uncertainties, methanol is the second most abundant molecule along the line of sight to W33A. Methanol has been detected in comets at the few percent level (Reuter 1992 and refs. therein).

A feature at $4.62 \mu \mathrm{m}$ in the spectrum of W33A has been ascribed to the $\mathrm{OCN}^{-}$ion. This species is formed by UV photolysis of laboratory ices containing $\mathrm{CO}$ and $\mathrm{NH}_{3}$ (Grim \& Greenberg 1987), possibly indicating 
that UV photolysis is significant in some regions in dense clouds. However, isonitriles such as $\mathrm{CH}_{3} \mathrm{NC}$ also produce a feature near $4.62 \mu \mathrm{m}$ and can not be excluded.

d'Hendecourt \& Jourdain de Muizon (1989) discovered an absorption feature at $15.2 \mu \mathrm{m}$ in the low resolution IRAS spectra of several embedded sources. Based on a comparison with laboratory data, they tentatively assigned this feature to solid $\mathrm{CO}_{2}$. Gerakines et al. (1995) found that solid $\mathrm{CO}_{2}$ has an abundance of $10 \%$ relative to water ice toward the embedded source GL 961. However, it is much less abundant towards other sources $(\leqslant 2 \%)$. Since $\mathrm{CO}_{2}$ is likely to be produced primarily by UV processing of mantles containing $\mathrm{CO}$ and $\mathrm{H}_{2} \mathrm{O}$, the apparent large variability in the $\mathrm{CO}_{2}$ abundance may reflect differences in the ambient $\mathrm{UV}$ fields.

Extensive laboratory experiments have been carried out over the last 15 years to understand the physical and chemical processes affecting evolution of grain mantles (e.g. Greenberg 1982, Grim et al. 1989; d'Hendecourt et al.1986; Tielens \& Allamandola 1987; Moore \& Donn 1982; Strazzulla \& Johnson 1991; Allamandola et al. 1988). Only a brief summary will be given here.

We have already discussed grain surface reactions with gas phase atoms in reducing or oxidizing environments. These reactions can lead to a $\mathrm{D} / \mathrm{H}$ ratio in the ices comparable to the gas phase ratio of $\sim 10^{-2}$ that results from ion-molecular gas phase chemistry.

Other chemical reactions can take place spontaneously when the accreted icy mantles are heated, producing new chemical species. For example, Schutte et al. (1993) have observed that, when $\mathrm{H}_{2} \mathrm{CO}$ and at least a trace of $\mathrm{NH}_{3}$ are present in the initial ice mixture, heating causes the $\mathrm{H}_{2} \mathrm{CO}$ to form chain polymers of polyoxymethylene (POM) and its derivatives.

The most important result of the experiments is the demonstration that UV photolysis or ion bombardment of low-temperature ice mixtures can lead to the formation of chemically complex organic residues that are stable when the sample is warmed to room temperature. The residue produced by UV photolysis of an ice mixture consisting of $\mathrm{H}_{2} \mathrm{O}, \mathrm{NH}_{3}$, and $\mathrm{CO}$ contains a variety of organic species with up to three $\mathrm{C}$ atoms. They are rich in $\mathrm{O}$ and $\mathrm{N}$, containing subgroups such as $-\mathrm{OH}, \mathrm{NH}_{2}$, and $\mathrm{C}=\mathrm{O}$ (Briggs et al. 1992). Perhaps such material is related to the abundant 
oxygen-rich organic species that were detected in Halley's coma (section $4.1)$.

For gas density $\sim 10^{4} \mathrm{~cm}^{-3}$, all the gas phase molecules (except $\mathrm{H}_{2}$ ) should accrete onto grain surfaces in $\sim 10^{5} \mathrm{yrs}$, a timescale short compared to the ages of molecular clouds. Since gaseous molecules are indeed observed, a mechanism to replenish the gas phase is needed. One possible process, supported by laboratory experiments, is explosive desorption of grain mantles (Schutte \& Greenberg 1991 and refs. therein). When $10 \mathrm{~K}$ ice mixtures are exposed to UV irradiation, potential chemical energy is produced, in the form of free radicals. When the ice is subsequently heated above $-26 \mathrm{~K}$, these radicals react explosively, releasing sufficient energy for desorption of volatile species such as $\mathrm{CO}$.

Several major issues concerning interstellar ices and organic solids need further investigation. The importance of UV and other energetic processing of the ices within dense clouds remains unclear. Future observations of the very strong $4.46 \mu \mathrm{m}$ feature of $\mathrm{CO}_{2}$, abundantly produced in the laboratory by photolysis of ices containing $\mathrm{CO}$ and $\mathrm{H}_{2} \mathrm{O}$, with the European Infrared Space Observatory (ISO) will shed new light on this issue. A related issue is the efficiency with which organic material is produced in the ice mantles. Sensitive high-resolution spectroscopy of the $5-8 \mu \mathrm{m}$ region as may be obtained by ISO is necessary to search for organic molecules that might be mixed into the ice mantles. Among others, this region contains the organic $\mathrm{C}=\mathrm{O}$ stretching mode at 5.8-6.0 $\mu \mathrm{m}$ and the $-\mathrm{CH}_{2}$ and $\mathrm{CH}_{3}$ deformation modes between 6.8-7.2 $\mu \mathrm{m}$. Finally, this region also contains the fingerprint features of the potentially important ice constituents $\mathrm{O}_{2}, \mathrm{CH}_{4}$, and $\mathrm{H}_{2} \mathrm{CO}$, as well as the sulfur containing species $\mathrm{SO}_{2}$ and $\mathrm{SO}_{3}$. Obtaining the abundances of these species and gaining some information on the surrounding ice matrix from the infrared bands will help to understand the chemical and physical conditions in dense clouds in which condensation of the icy mantles took place.

\subsection{Evolution of Dust in Protoplanetary Disks}

Accretion disks created by infalling material from the protostellar envelope are now considered to be a natural part of the evolution of a solarmass star (section 2.1). Disk masses are typically 0.01-0.1 $\mathrm{M}_{0}$ and disk 
sizes are $\lesssim 100 \mathrm{AU}$. An important factor in the subsequent evolution of protoplanetary disks is the presence or absence of turbulence. The most likely cause of turbulence is a superadiabatic vertical temperature gradient, leading to vertical turbulent convection. Such a steep thermal gradient is due to opacity, which inhibits radiative heat flow.

Although dust is a small fraction of the total nebular mass, it exerts a strong influence on the evolution of the nebula, because dust is the source of the opacity and the opacity determines whether thermal convection will occur. Thus, knowledge of the dust optical properties is important. Yet, the composition and size distribution of the initial dust population feeding the accretion disk are not well known (section 5.1). Rosseland mean opacities have been computed by Pollack et al. (1994) and Preibisch et al. (1993) among others.

The opacity will increase as grains begin to grow to a size comparable to the wavelength, causing an increase in turbulent convection. Turbulence generates size-dependent differential dust velocities leading to grain-grain collisions and rapid grain growth, as larger particles sweep up smaller ones. (If the collision velocities are too high, grains can be shattered or vaporized.) Coagulation may produce large fractal aggregates. Aggregates with an open structure will tend to have the optical properties of their structural components; if the components have size comparable to the wavelength, the opacity will be maximum. Thus, turbulence accelerates grain growth and grain growth increases opacity, creating stronger convective turbulence. This tight coupling between turbulence and grain growth has to be addressed in any realistic accretion disk model (e.g. Mizuno 1989, Sterzik \& Morfill 1994, and refs. therein).

Opacity, hence turbulence, is not necessarily uniform - if the disk is still accreting small grains from the envelope, for example (Mizuno 1989). Sterzik \& Morfill (1994) consider the effect of condensation regions (e.g. water ice) on the radial profile of turbulence. They conclude that lower turbulence and enhanced surface density can occur in condensation zones, perhaps related to the formation of the giant planets.

Although turbulence fosters grain growth, it prevents the dust from settling to the central plane. A critical stage is reached when grains have grown large enough (size $>>\lambda$ ) that opacity decreases and the nebula becomes optically thin. Then the nebula can cool by radiation and thermally-driven turbulence ceases. The dust particles, which have already 
grown to mm-cm size, rapidly settle to the mid-plane, forming a thin dust layer in which Goldreich-Ward (1973) gravitational instability can develop, leading to the growth of $\mathrm{km}$-sized planetesimals.

Miyake \& Nakagawa (1994) have modeled the temperature distribution and infrared spectral energy distribution (SED) of passive thin disks and flaring disks. They can fit the SED of many T Tauri stars and can distinguish between stars that fit the thin disk and flaring disk models, which they conclude is evidence that dust has settled to a thin plane in a subset of T Tauri stars.

One important question is the extent that dust can be radially transported in the disk from the warm inner nebula at $\mathrm{R} \leq 1 \mathrm{AU}$ to the region of come formation at $\mathrm{R}>10$ AU. Cuzzi et al. (1993) have computed that small grains entrained in the outflowing gas near the midplane of the solar nebula could drift radially outward 2-5 AU before being accreted onto larger particles. Grain growth was a rapid process (Weidenschilling 1988; Mizuno 1989), limiting the time available for radial diffusion.

In summary, modeling the evolution of protoplanetary disks is an active research area at the present time. Some of the open questions relevant to the evolution of the dust are:

1. What is the composition and initial size distribution of dust in accretion disks, and what is the corresponding opacity?

2. Is thermal convection the sole cause of turbulence?

3. What effect did species condensation, such as water ice, have on the opacity and subsequent evolution of the nebula?

4. To what extent were dust particles transported radially? Was radial transport sufficient to bring inner solar system condensates to the zone of comet formation?

\subsection{Evolution of Dust Properties from Molecular Clouds to Comets}

We have seen that the composition, size, and shape of IS grains are strongly modified within molecular clouds. Although models differ in detail, there is a general picture of silicate cores with accreted mantles in various stages of processing, from organic refractory residues to an outer 
layer of volatile ices. These core-mantle particles, as well as small bare silicate and carbon grains, are agglomerated into fluffy structures.

These heterogenous particles constituted the solid portion of the material from which the protosolar nebula evolved. As the protosolar envelope collapsed to form an accretion disk and a growing central star, the solid particles were heated and the more volatile components sublimated, leaving a dust composition that varied with distance from the protosun.

Current evidence tells us that comets formed cold, in the outer parts of the solar nebula where accretion shock heating and radiant heating from the protosun were minimal. How cold has an important effect on the volatile species in the grains, and there could be a considerable, gradient over the region of comet formation from 12 to $50 \mathrm{AU}$. Water ice, however, should be stable over this entire region.

To what extent do comets consist of unaltered molecular cloud dust? Let us examine 3 dust components: refractory (silicates), organic material, and ices.

The silicate feature in several comets shows an $11.2 \mu \mathrm{m}$ peak due to crystalline olivine, superimposed on a broad smooth feature peaking at $9.8 \mu \mathrm{m}$ (section 3.1). The broad feature resembles that seen in both the diffuse ISM and dense clouds. With the exception of a weak signature in AFGL2591 (Aitken et al. 1988), the $11.2 \mu \mathrm{m}$ olivine peak has not been detected in any protostellar source (Hanner et al. 1995 and refs. therein) nor in the IS medium. Thus, a fraction of the silicate material in comets is apparently not of IS origin. Crystalline olivine condenses at T 1400 K. It can also form by annealing from glassy silicate at $T \geq 875 \mathrm{~K}$. Thus, we are apparently seeing evidence of a high temperature component formed in the inner solar system and transported to the region of comet formation.

This raises an important issue: When considering the organic components, we often assume that the comet material is "local", i.e., not heated within the solar system. But, if there is a component of refractory silicates transported from the inner solar nebula, there may also be a higher-temperature organic component transported to the comet-forming region as well.

Organic material is thought to be the main reservoir of carbon in comets (Greenberg \& Shalabiea 1993). The dust impact experiment on the Halley probes proved that refractory organic material ("CHON") is an important component of comet dust. These grains are apparently the 
source of $\mathrm{CO}, \mathrm{H}_{2} \mathrm{CO}$, and other gaseous species in the coma requiring a distributed source (e.g. Meier et al. 1993). High D/H ratios approaching the IS value, are associated with carbon-bearing materials in meteorites and IDPs (section 4.3). Polymerized $\mathrm{H}_{2} \mathrm{CO}$, and similar long chain polymers can explain the pattern of heavy atomic mass species detected by the gas mass spectrometers on the Halley probes (Korth et al. 1986). Such polymers are a product of photolysis of icy grain mantles in molecular clouds (Schutte et al. 1993). Cometary spectra show an "organic" feature near $3.36 \mu \mathrm{m}$, but at least part of the feature is due to gas phase methanol, and the remainder may also arise from a gaseous species (section 4.1).

Water ice comprises a significant fraction of cometary material. Was the water ice initially incorporated into comets amorphous or crystalline? The amorphous to crystalline phase change at $130-140 \mathrm{~K}$ has often been invoked as an energy source to explain cometary outbursts at large heliocentric distance. Kouchi et al. (1994) have examined the criteria for formation and preservation of amorphous water ice in molecular clouds and in the solar system. Whether amorphous or crystalline ice forms depends upon the $\mathrm{H}_{2} \mathrm{O}$ deposition rate as well as temperature. They conclude that amorphous ice will form in dense clouds and that IS icy grains will remain amorphous over the $\sim 10^{8} \mathrm{yr}$ lifetime of the solar nebula at $\mathrm{R} \gtrsim 12 \mathrm{AU}$. However, they predict that ice which formed in the solar nebula will be crystalline.

The $\mathrm{CO}: \mathrm{H}_{2} \mathrm{O}$ abundance in comets is a strong argument that cometary ices stem directly from IS ices, not from the gas phase. In the gas phase of dense clouds, $\mathrm{CO}: \mathrm{H}_{2} \mathrm{O}$ is 10:1 or larger, while in the gases sublimating from comets, $\mathrm{CO}: \mathrm{H}_{2} \mathrm{O}$ is about $1: 5$ or less, a factor of 50 difference. Molecules such as $\mathrm{H}_{2} \mathrm{CO}, \mathrm{CH}_{3} \mathrm{OH}, \mathrm{CO}_{2}$, which are present at the few percent level in comets, are UV photolysis products in IS grain mantles.

Thus, in general, the relative abundances of molecular species in comets are consistent with the inferred (observed or predicted) composition of IS dust in dense regions (e.g. Greenberg \& Shalabiea 1993). Information about the structure of cometary dust comes from interplanetary dust particles (IDPs; section 3.2). In the $10 \mu \mathrm{m}-50 \mu \mathrm{m}$ size range sampled by stratospheric collections, the IDPs of likely cometary origin are fluffy aggregates. Did these large aggregate particles form in the cometary nucleus, the solar nebula, or the parent molecular cloud? A major 
component consists of $0.1-0.3 \mu \mathrm{m}$ glassy silicate grains with sputtered rims; these appear to be interstellar grains (Bradley 1994). These grains are embedded in a carbonaceous material, which could be IS organicrefractory residues. Yet, the aggregates also contain high-temperature condensates, such as micron-sized pyroxene whiskers and platelets that argue for a solar nebula origin. A minor amount of hydrated silicate is also present.

Thus, the heterogeneous mix of materials in IDPs suggests that the large aggregates formed in the solar nebula, incorporating IS grains and solar system condensates. Aggregation could also have occurred during formation of the comet nuclei, wherein the grains were mixed with ices. The fluffy structure, then, would result from the sublimation of ices that originally filled the pores.

In summary, the evidence from comets and cometary IDPs is consistent with their formation primarily from IS dust similar to that seen in dense clouds, including preservation of low-temperature ices. Some high-temperature solar system material may have been added during cometary formation.

\section{ACKNOWLEDGEMENTS}

This summary is based upon the invited presentations by $\mathrm{Ph}$. Andre: Protoplanetary disks around pre-main-sequence objects; Th. Henning: Optical properties of dust in dense regions; D. Backman: The dust in $\beta$ Pictoris systems; A. C. Levasseur-Regourd: Optical/infrared properties of cometary and interplanetary dust; S. Sandford: What can interplanetary dust particles tell us about interstellar dust?; E. Zinner: Interstellar dust in primitive meteorites; M. Rowan-Robinson: Properties of interstellar dust from IRAS observations; P. Martin: Optical properties of interstellar dust in diffuse and molecular clouds; J. M. Greenberg: Evolution of the dust properties from molecular clouds to comets; W. Schutte: Evolution of ices and organic compounds; Y. Nakagawa: Evolution of grains in protoplanetary systems. I would like to thank all of the speakers, colleagues who contributed poster papers, and the S.O.C.: H. Habing, P. Martin, J. Mathis, and H. J. Völk. M. Hanner's research is supported at the Jet Propulsion Laboratory, California Institute of Technology, under contract with the National Aeronautics and Space Administration. 


\section{REFERENCES}

Adams, F.C., Lada, C.J. \& Shu, F.H. 1987. ApJ 312, 788.

Agladze, N.I., Sievers, A.J., Jones, S.A., Burlitch, J.M. \& Beckwith,

S.V.B. 1994. ApJ, in press.

Aitken, D.K. Roche, P.F., Smith, C.H., James, S.D. \& Hough, J.H. 1988. MNRAS 230, 629.

Allamandola, L.J., Sandford, S.A., Tielens, A.G.G.M. \& Herbst, T.M. 1992. ApJ 399, 134.

Allamandola, L.J., Sandford, S.A., Tielens, A.G.G.M., \& Herbst, T.M. 1993. Science 260, 64.

Allamandola, L.J., Sandford, S. A. \& Valero, G. J. 1988. Icarus 76, 225. Allamandola, L.J., Tielens, A.G.G.M., \& Barker, J.R. 1985. ApJ 290, 25. Anders, E. \& Zinner, E. 1993. Meteoritics 28, 490.

André, Ph. \& Montmerle, T. 1994. ApJ 420, 837.

André, Ph., Ward-Thompson, D. \& Barsony, M. 1993. ApJ 406, 122.

Appenzeller, I., Jankovics, I. \& Ostreicher, R. 1984. A\&A 141, 108.

Backman, D. \& Paresce, F. 1992. In Protostars and Planets III, ed. E.H.

Levy, J.I. Lunine, \& M.S. Matthews, Univ. Arizona Press, Tucson, p. 1253.

Bastien, P. \& Menard, F. 1988. ApJ 326, 334.

Bastien, P. \& Menard, F. 1990. ApJ 364, 232.

Beckwith, S.V.W. \& Sargent, A.I. 1991. ApJ 381, 250.

Beckwith, S.V.W., Sargent, A.I., Chini, R.S. \& Güsten, R. 1990. AJ 99, 924.

Beust, H., Lagrange-Henri, A.M., Vidal-Madjar, A. \& Ferlet, R. 1990. A\&A 236, 202.

Boulanger, F. Beichman, C., Desert, F.X., Helou, G., Perault, M., \& Ryter, C. 1988. ApJ 332, 328.

Boulanger, F. \& Perault, M. 1988. ApJ 330, 964.

Bradley, J.P. 1994. Science 265, 925-29.

Bradley, J.P., Humecki, H.J. \& Germani, M.S. 1992 ApJ 394, 643. Bradley, J.P., Sandford, S.A. and Walker, R.M. 1988. In Meteorites and the Early Solar System, ed. J. Kerridge \& M. Matthews, Univ. Arizona Press, Tucson, p. 861. 
Briggs, R., Ertem, G., Ferris, J.P., Greenberg, J.M., McCain, P.J., Mendoza-Gomez, C.X. \& Schutte, W. 1992. In Origins of Life and Evolution of the Biosphere 22, 287.

Cuzzi, J. N., Dobrovolskis, A. R. \& Champney, J. M. 1993. Icarus 106, 102.

d'Hendecourt, L.B., Allamandola, L.J., Grim, R.J.A. \& Greenberg, J.M. 1986. A\&A 158, 119.

d'Hendecourt, L.B. \& Jourdain de Muizon, M. 1989. A\&A 223, L5.

Davies, J. , Green, S.F. \& Geballe, T.R. 1991. MNRAS 251, 148.

Divine, N. 1993. Jour. Geophys. Res. 98, 17029.

Dollfus, A., Bastien, P., Le Borgne, J.-F., Levasseur-Regourd, A.C. \& Mukai, T. 1988. A\&A 206, 348.

Draine, B. 1989. In Interstellar Dust, ed. L.J. Allamandola \& A.G.G.M. Tielens, Kluwer, Dordrecht, p. 313.

Draine, B.T. \& Lee, H.M. 1984. ApJ 285, 89.

Draine, B.T. \& Malhotra, S. 1993. ApJ 414, 632.

Duley, W.W. \& Williams, D.A. 1981. MNRAS 196, 269.

Duley, W.W. 1987. MNRAS 229, 203.

Duncan, M., Quinn, S. \& Tremaine, S. 1988. ApJ Lett. 328, L69.

Eberhard, P. et al. 1987. A\&A 187, 435.

Edwards, S., Cabrit, S., Strom, S.E., Heyer, I., Strom, K.M. \& Anderson, E. 1987. ApJ 321, 473.

Geballe, T.R. 1986. A\&A 162, 348.

Gerakines, P.A., Schutte, W.A., Greenberg, J.M. \& van Dishoeck, E.F. 1995. A\&A, in press.

Goldreich, P. \& Ward, W. R. 1973. ApJ 183, 1051.

Greenberg, J.M. 1989. In Interstellar Dust, ed. L.J. Allamandola \& A.G.G.M. Tielens, Kluwer Dordrecht, 1989, p. 345.

Greenberg, J.M. 1982. In Comets, ed. L.L. Wilkening, Univ. Arizona Tucson, p. 131.

Greenberg, J.M. \& Shalabiea, O.M. 1993. In Asteroids, Comets, Meteors 1993, ed. A. Milani, M. Di Martino \& A. Cellino, Kluwer Dordrecht, p. 327.

Grim, R.J.A., Baas, F., Geballe, T.R., Greenberg, J.M. \& Schutte, W. 1991. A\&A 243, 473.

Grim, R.J.A. \& Greenberg, J.M. 1987. ApJ 321, L91. 
Grim, R.J.A., Greenberg, J.M., de Groot, M.S., Baas, F., Schutte, W.A. \& Schmitt, B. 1989. A\&A Suppl. 78, 161.

Grün, E. et al. 1994. A\&A 286, 915.

Grün, E. Zook, H.A., Fechtig, H. \& Giese, R.H. 1985. Icarus 62, 244.

Hanner, M.S., Brooke, T.Y. \& Tokunaga, A.T. 1995. ApJ 438, in press. Hanner, M.S., Lynch, D.K. \& Russell, R.W. 1994a. ApJ. 425, 274.

Hanner, M.S., Hackwell, J.A., Russell, R.W. \& Lynch, D.K. 1994 b. Icarus 112 , in press.

Hayashi, M., Ohashi, N. \& Miyama, S.M. 1993. ApJ 418, L71.

Henning, Th., Pfau, W., Zinnecker, H. \& Prusti, T. 1993. A\&A 276, 129. Hildebrand, R.H. 1983. Quar. Jour. Roy. Ast. Soc. 24, 267.

Huffman, D.R. 1987. In Proc. Capri Workshop on Cosmic Dust Analogues, Capri, Sept. 9-12 1987.

Jenkins, E. 1989. In Interstellar Dust, ed. L.J. Allamandola \& A.G.G.M. Tielens, Kluwer Dordrecht, p. 23.

Jessberger, E.K., Christoforidis, A. \& Kissel, J. 1988. Nature 332, 691.

Jones, A.P., Tielens, A.G.G.M., Hollenbach, D.J., \& McKee, C.F. 1994. ApJ 433, 797.

Keene, J. \& Masson, C.R. 1990. ApJ 355, 635.

Kissel, J. et al. 1986. Nature 321, 280; Nature 321, 336.

Knacke, R.F., Fajardo-Acosta, S.B., Telesco, C.M., Hackwell, J.A., Lynch, D.K., and Russell, R.W. 1993. ApJ 418, 440.

Knacke, R.F. \& McCorkle, S.M. 1987. AJ 94, 972.

Koike, C., Hasegawa, H. \& Manabe, A. 1980. Ap. Space Sci. 67, 495. Korth, A. et al. 1986. Nature 321, 335.

Kouchi, A., Yamamoto, T., Kozasa, T. Kuroda, T. \& Greenberg, J. M. 1994. A\&A 290, 1009.

Lacy, J.H., Baas, F., Allamandola, L.J., van de Bult, C., Persson, S.E., McGregor, P.J., Lonsdale, C.J. \& Geballe, T.R. 1984. ApJ 276, 533.

Lada, C.J. 1987. In IAU Symp. 115, Star Forming Regions, ed. M.

Peimbert \& J. Jugaku (Dordrecht: Kluwer), p. 1.

Leger, A., Gauthier, S., Defourneau, D. \& Rouan, D. 1983. A\&A 117, 164.

Leger, A. \& Puget, J.L. 1984. A\&A 137, L6.

Levasseur-Regourd, A.C., Dumont, R., \& Renard, J.B. 1990. Icarus 86, 264. 
Levasseur-Regourd, A.C. \& Hadamcik, E. 1994. Plan. Space Sci., in press. Mathis, J.S. 1994. ApJ 422, 176.

Mathis, J.S., Rumpl, W. \& Nordsieck, K.H. 1977. ApJ 217, 425.

Mathis, J.S. \& Whiffen, G. 1989. ApJ 341, 808.

McKee, C. 1989. In Interstellar Dust, ed. L.J. Allamandola \& A.G.G.M. Tielens, Kluwer, Dordrecht, p. 431.

McKeegan, K.D., Walker, R.M. \& Zinner, E. 1985. Geochim.

Cosmochim. Acta 49, 1971-87.

Meier, R., Eberhardt, P., Krankowsky, D. \& Hodges, R. R. 1993. A\&A $277,677$.

Miyake, K. \& Nakagawa, Y. 1994. ApJ, in press.

Mizuno, H. 1989. Icarus 80, 189.

Moore, M.H. \& Donn, B. 1982. ApJ Lett. 257, L47.

O'Dell, C.R. \& Wen, Z. 1994. ApJ 436, 194.

Page, L.A., Cheng, E.S. \& Meyer, S.S. 1990. ApJ Lett. 355, L1.

Palumbo, M.E. \& Strazzula, G. 1993. A\&A 269, 568.

Pollack, J.B., Hollenbach, D., Beckwith, S., Simonelli, D.P., Roush, T. \& Fong, W. 1994. ApJ 421, 615.

Preibisch, Th., Ossenkopf, V., Yorke, H.W. \& Henning, Th. 1993. A\&A $279,577$.

Reuter, D.C. 1992. ApJ 386, 330.

Rowan-Robinson, M. 1992. MNRAS 258, 787.

Rowan-Robinson, M. 1986. MNRAS 219, 737.

Sakata, A., Wada, S., Onaka, T. \& Tokunaga, A.T. 1987. ApJ 320, L63.

Sandford, S.A., Allamandola, L.J., Tielens, A.G.G.M. \& Valero, G.J. 1988. ApJ 329, 498.

Sandford, S.A. \& Walker, R.M. 1985. ApJ 291, 838.

Sargent, A.I. \& Beckwith, S. 1987. ApJ 323, 294.

Sargent, A.I. \& Beckwith, S. 1991. ApJ 382, L31.

Savage, B.D., Cardelli, J.A. \& Sofia, U.J. 1992. ApJ 401, 706

Schutte, W.A., Allamandola, L.J. \& Sandford, S.A. 1993. Icarus 104, 118.

Schutte, W.A. \& Greenberg, J.M. 1991. A\&A 244, 190.

Schutte, W.A., Tielens, A.G.G.M. \& Sandford, S.A. 1991. ApJ 382, 523.

Seab, C.G. \& Shull, J.M. 1986. In Interrelationships among

Circumstellar, Interstellar, and Interplanetary Dust, NASA Conf. Pub. 2403, p. 37. 
Sellgren, K. 1989. In Interstellar Dust, ed. L.J. Allamandola \& A.G.G.M. Tielens, Kluwer Dordrecht, p. 103.

Sellgren, K. 1984. ApJ 277, 623.

Skinner, C.J., Tielens, A.G.G.M., Barlow, M.J. \& Justtanont, K. 1992. ApJ 399, L79.

Skrutskie, M.F., Dutkevitch, D., Strom, S.E., Edwards, S. \& Strom, K.M. 1990. Astron. J. 99, 1187.

Smith, B.A. \& Terrile, R.J. 1984. Science 226, 1421.

Smith, B.A. \& Terrile, R.J. 1987. Bull AAS 19, 829.

Sofia, U.J., Cardelli, J.A. \& Savage, B.D. 1994. ApJ 430, 650.

Sofia, U.J., Savage, B.D. \& Cardelli, J.A. 1993. ApJ 413, 251.

Sorrell, W.H. 1990. MNRAS 243, 570.

Spitzer, L. 1985. ApJ Lett. 290, L21.

Sterzik, M. F. \& Morfill, G. E. 1994. Icarus 111, 536.

Strazzulla, G. \& Johnson, R.E. 1991. In Comets in the Post-Halley Era, ed. R.L. Newburn, M. Neugebauer \& J. Rahe, Kluwer, Dordrecht, p. 243.

Strom, S.E., Edwards, S. \& Skrutskie, M.F. 1993. In Protostars and Planets III, ed. E.H. Levy and J.I. Lunine, Univ. Arizona Press, Tucson, p. 837.

Sykes, M.V. \& Walker, R.G. 1992. Icarus 95, 180.

Tereby, S., Chandler, C.J. \& André, Ph. 1993. ApJ 414, 759.

Tereby, S., Shu, F.H. \& Cassen, P. 1984. ApJ 286, 529.

Tielens, A.G.G.M. 1989. In IAU Symposium 135, Interstellar Dust, ed.

L.J. Allamandola \& A.G.G.M. Tielens Kluwer, Dordrecht, p. 239. Tielens, A.G.G.M. \& Allamandola, L.J. 1987. In Physical Processes in Interstellar Clouds, ed. G.E. Morfill \& M. Scholer, Reidel, Dordrecht, p. 333.

Tielens, A.G.G.M., Tokunaga, A.T., Geballe, T.R. \& Baas, F. 1991. ApJ $381,181$.

Vrba, F.J., Coyne, G.V. \& Tapia, S. 1993. AJ 105, 1010.

Vrba, F.J., Coyne, G.V. \& Tapia, S. 1981. ApJ 243, 489.

Weidenschilling, S. J. 1988. In Meteorites and the Early Solar System, ed. J. F. Kerridge \& M. S. Matthews, Univ. Arizona Press, Tucson, p. 348.

Whittet, D.C.B., Duley, W.W. \& Martin, P.G. 1990. MNRAS 244, 427. Wilking, B.A., Lada, C.J. \& Young, E.T. 1989. ApJ 340, 823. 
Wright, E.L., 1987. ApJ 320, 818.

Zinner, E. 1988. In Meteorites and the Early Solar System, ed. J.F.

Kerridge \& M.S. Matthews, Univ. Arizona Press, Tucson, p. 956. Zuckerman, B. \& Becklin, E.E. 1993. ApJ 414, 793. 